\title{
From Aluminum Foil to Two-Dimensional Nanocrystals Using Ultrasonic Exfoliation
}

\author{
Weigang Lu,* Blake Birmingham, Dmitri V. Voronine, Drew Stolpman, Sharad Ambardar, \\ Deniz Altunoz Erdogan, Emrah Ozensoy, Zhenrong Zhang,* and Touradj Solouki*
}

Cite This: J. Phys. Chem. C 2021, 125, 7746-7755

Read Online

ACCESS

Llll Metrics \& More

Article Recommendations

Supporting Information

ABSTRACT: Al nanostructures have unique optical properties such as widely tunable surface plasmon resonances from deep UV to NIR that can be used for label-free fluorescence enhancement and surface-enhanced Raman scattering. Various $\mathrm{Al}$ nanostructures have been fabricated using sophisticated "top-down" lithographic and "bottom-up" colloidal methods. Here, we developed a simple and efficient method of synthesizing two-dimensional (2D) aluminum (Al) nanocrystals from commercially available $\mathrm{Al}$ foil using

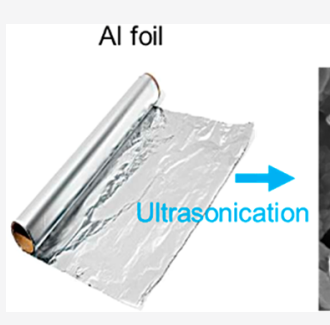
$2 \mathrm{D}$ nanocrystals Enhanced Raman ultrasonic exfoliation under ambient environment. 2D $\mathrm{Al}$ nano-

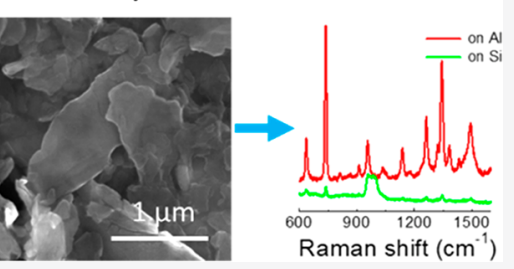
crystals with sizes from a few hundred nanometers to several micrometers and thickness in the tens of nanometers were isolated through centrifugation separation. The exfoliated $2 \mathrm{D} \mathrm{Al}$ nanocrystals are covered with a passivated $\mathrm{Al}_{2} \mathrm{O}_{3}$ nanolayer. The determined exfoliation mechanism is a combination of the preferred cleavage along the (111) surface planes and layer-by-layer $\mathrm{Al}_{2} \mathrm{O}_{3}$ exfoliation from the surface of the $2 \mathrm{D} \mathrm{Al}$ nanocrystals. We demonstrate that the $2 \mathrm{D}$ Al nanocrystals can be assembled at water/air interface and transferred to different substrates to form $2 \mathrm{D} \mathrm{Al}$ nanocrystal films. These $2 \mathrm{D}$ Al nanocrystal films exhibit surface plasmon resonance in the visible spectral range and show enhanced Raman signals of adenine using a $532 \mathrm{~nm}$ excitation. These $2 \mathrm{D}$ Al nanocrystal films could be further developed for new optical and sensing applications.

\section{INTRODUCTION}

Owing to their wide range of applications in various fields of science, metallic nanostructures with surface plasmon resonances (SPRs) have been studied extensively. ${ }^{1,2}$ SPRs can be selectively tuned in the UV-vis-NIR range by varying the size and shape of the nanostructures and by modifying their surfaces. Most SPR studies focus on development and characterization of $\mathrm{Au}$ and $\mathrm{Ag}$ nanostructures due to their strong field enhancement in the visible range and ease of fabrication. However, other metals, such as aluminum, have SPR properties that can provide beneficial spectroscopic advantages. For example, Al nanostructures' SPRs can be tuned from deep UV to NIR by controlling the nanoparticle size and surface oxide thickness. ${ }^{3-5}$ Moreover, aluminum is the most abundant metal in the earth's crust and the most widely used metal next to iron. Therefore, it would be useful to utilize $\mathrm{Al}$ nanostructures for optical sensing and imaging applications. Most reported plasmonic $\mathrm{Al}$ nanostructures including $\mathrm{Al}$ thin films, ${ }^{6,7}$ nanoparticles, ${ }^{4,8-12}$ and nanovoids ${ }^{13,14}$ have been fabricated using deposition coupled with lithography. Recently, Halas's group reported a breakthrough in the synthesizing of $\mathrm{Al}$ nanocolloids, using a "bottom-up" approach in an argonprotected environment, and confirmed that $\mathrm{Al}$ nanocolloids have tunable optical resonance which shows great potential in surface-enhanced Raman applications for pollutant detection and photocatalytic reactivities. ${ }^{15-22}$
Two-dimensional (2D) materials have generated great interest due to their unique electrical and optical properties. Thousands of compounds with the intrinsic layered structure have been predicted that could be exfoliated to $2 \mathrm{D}$ structures, $^{23,24}$ while nonlayered materials are not regarded as candidates for $2 \mathrm{D}$ materials. ${ }^{25}$ Unlike layered $2 \mathrm{D}$ materials, metals usually have close-packed structures and have different numbers of slip planes according to their lattice structures. Recently, a new method of cryogenic exfoliation of $\mathrm{Mg}$ into 2D nanocrystals through ultrasonication has been reported. ${ }^{25}$ In this method, liquid nitrogen treatment is employed to change the slip system in magnesium. Metals such as aluminum, copper, and nickel have good ductility because of their abundant slip planes in their face-centered cubic (FCC) crystals. The abundance and ductility of aluminum makes $\mathrm{Al}$ foil the most widely used metal in household and commercial packaging. Al foil is produced by rolling $\mathrm{Al}$ ingots into the desired thickness through continuous casting or cold rolling. Therefore, Al foil consists of layers of highly elongated,

Received: December 7, 2020

Revised: $\quad$ March 24, 2021

Published: April 2, 2021 


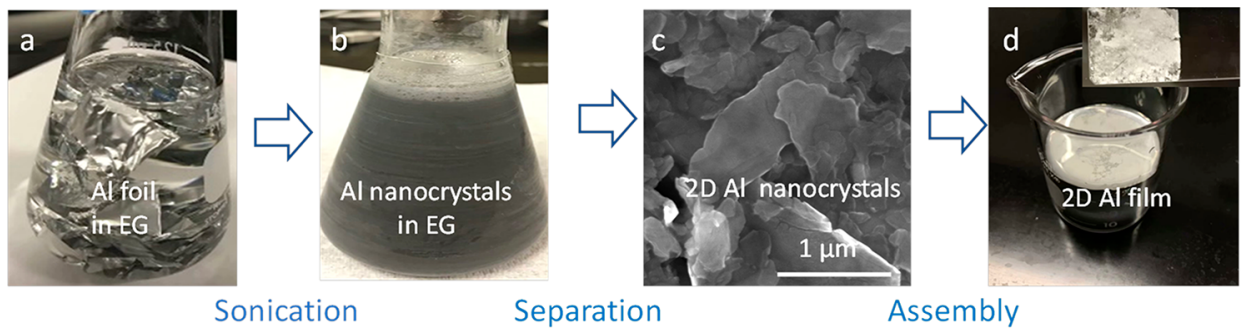

Figure 1. Al nanocrystal synthesis. (a) Picture of $\mathrm{Al}$ foil in EG solution before ultrasonication; (b) picture of $2 \mathrm{D}$ Al nanocrystals in EG after ultrasonication; (c) SEM image of separated 2D nanocrystals; (d) 2D Al nanocrystals assembled into film on water/air surface (inset shows a representative $2 \mathrm{D} \mathrm{Al}$ film transferred to a quartz substrate).

deformed grains. Ultrasonication is a simple but efficient method for wet-milling and microgrinding of particles with sizes from $\sim 10 \mathrm{~nm}$ to $500 \mu \mathrm{m} .^{26,27}$ Ultrasonic cavitation occurs when an ultrasound wave crosses a liquid in alternating highpressure and low-pressure cycles. At high sonication intensity, transient bubbles form and expand through a few acoustic cycles before they collapse. The temperature and pressure of these collapsing bubbles can be as high as several thousand degrees and a thousand atmospheres, respectively. ${ }^{28-31}$ The implosion event, when occurring near a hard surface, changes the bubble into a jet about one-tenth the bubble size, which travels at speeds up to $400 \mathrm{~km} / \mathrm{h}$ toward the hard surface. ${ }^{32}$ Ultrasonic cavitation is the basis of ultrasound cleaning and milling. ${ }^{27}$ Immersing a piece of $\mathrm{Al}$ foil in an ultrasonicated liquid can yield a perforated and wrinkled foil; therefore, $\mathrm{Al}$ foil tests have been used to demonstrate the power distribution in ultrasonic devices. ${ }^{27}$ In addition, $\mathrm{Al}$ particles with the sizes in tens of micrometers have been produced when $\mathrm{Al}$ foil is ultrasonicated in water. ${ }^{33}$ Previously, we reported that $2 \mathrm{D} \mathrm{Al}$ nanocrystals made from commercially available $\mathrm{Al}$ foil through ultrasonication can be used as nanoshields for improved radiation-based bacterial treatments. ${ }^{34}$ In this work, we studied the mechanism for converting $\mathrm{Al}$ foil into $2 \mathrm{D} \mathrm{Al}$ nanocrystals by examining the structural evolution of $\mathrm{Al}$ foil and $2 \mathrm{D} \mathrm{Al}$ nanocrystals during the ultrasonic exfoliation process. We also demonstrate that the $2 \mathrm{D} \mathrm{Al}$ nanocrystals can be assembled at the water/air interface and transferred to different substrates forming $2 \mathrm{D} \mathrm{Al}$ nanocrystal films. The $2 \mathrm{D} \mathrm{Al}$ nanocrystal films exhibit surface plasmon resonance in the visible spectrum range and show enhanced Raman signals using a $532 \mathrm{~nm}$ laser excitation for adenine detection, making them of interest for optical and sensing applications.

\section{EXPERIMENTAL METHODS}

Standard commercial aluminum foil with a thickness of $\sim 16$ $\mu \mathrm{m}$ for home usage (Reynolds Wrap) was purchased from a local grocery store. Ethylene glycol (EG, analytical grade), methanol (HPLC grade), ethanol, glycerol (analytical grade), n-propanol (analytical grade), and adenine (99\%) were purchased from VWR (Radnor, PA, USA) and used without further purification. Water used in the experiments was NanoPure (18.2 M $\Omega$ ) generated by Barnstead nanopure water systems (Thermo Scientific, MA, USA).

In a typical ultrasonic exfoliation process, $0.16 \mathrm{~g}$ of $\mathrm{Al}$ foil was cut into small pieces and then put into $100 \mathrm{~mL}$ of ethylene glycol (Figure 1a) in an Erlenmeyer flask. The mixture was put into an ultrasonic cleaner (Branson 2510, Branson Ultrasonic, CT, USA) for ultrasonic exfoliation. During the ultrasonication, the $\mathrm{Al}$ foil gradually fragmented into smaller pieces, and the color of the mixture slowly changed from a colorless to grayish black (Figure 1b). The mixture was left on the countertop for an hour to settle down after sonication. The upper layer mixture from this solution was centrifuged at 2000 rpm for $10 \mathrm{~min}$ to separate the $\mathrm{Al}$ microparticles, followed by centrifugation at higher centrifugation speeds (10000-20000 $\mathrm{rpm}$ ) for $20 \mathrm{~min}$ to obtain a gray precipitation that contained the $2 \mathrm{D}$ aluminum nanoflakes (Figure 1c). 2D Al nanocrystals with different sizes can be separated by simply choosing different centrifugation speeds. The aluminum particles were washed with ethanol to remove any remaining ethylene glycol and solvent components. The purified samples were stored in anhydrous ethanol. The $\mathrm{Al}$ nanocrystals can assemble into $2 \mathrm{D}$ $\mathrm{Al}$ nanocrystal films on the water/air interface when the $\mathrm{Al}-$ ethanol mixture is carefully transferred onto a water surface dropwise as ethanol evaporated (Figure 1d). The Al films were then transferred onto different substrates (Figure 1d insert) for further characterization and application investigations.

The X-ray diffraction (XRD) patterns of the $2 \mathrm{D} \mathrm{Al}$ nanocrystals and $\mathrm{Al}$ foil were collected with a Siemens D5000 diffractometer in $\theta-2 \theta$ mode using $\mathrm{Cu} \mathrm{K} \alpha$ radiation $(\lambda=1.5406 \AA$ ) as the X-ray source (operated at $40 \mathrm{kV}$ and 30 A). The scanning step size for XRD was 0.05 degree.

Scanning electron microscope (SEM) images were collected with a FEI Focused Ion Beam SEM Microscope (FEI-Versa 3D). The energy-dispersive X-ray spectroscopy (EDS) experiments were performed to determine the composition of the commercial Al foil. The results show that commercial $\mathrm{Al}$ foil has $98.4 \% \mathrm{Al}, 0.6 \% \mathrm{Si}, 0.9 \% \mathrm{Fe}$, and negligible amounts of $\mathrm{Mn}$, $\mathrm{Zn}$, and $\mathrm{Cu}$ (Figure S1). Low magnification transmission electron microscopy (TEM) images were acquired with a JEOL 1010 TEM (JEOL Co., Japan) with $60 \mathrm{kV}$ accelerating voltage. High resolution TEM images were collected using a FEI Tecnai G2 F30 (FEI, USA) operated at $300 \mathrm{kV}$. Atomic force microscope (AFM) images were acquired using a Dimension Icon AFM microscope (Bruker Corp).

Raman measurements were conducted using a lab-built Raman microscope, which consisted of a $532 \mathrm{~nm}$ laser as the excitation source and a Zaber ASR-E closed loop microscope stage (Zaber Technologies Inc., Canada) for scanning and focusing control. A focused laser beam with a spot size of $\sim 1.0$ $\mu \mathrm{m}$ was scanned over the samples' surface. The Raman spectra were collected using a Princeton Instruments SCT-320 spectrometer with a 1200 lines/mm grating through a $60 \times$ objective lens. Adenine detection on the $\mathrm{Al} 2 \mathrm{D}$ nanocrystal film and $\mathrm{Si}$ substrate was accomplished by drop-casting a $3 \mu \mathrm{L} 10^{-4}$ $\mathrm{M}$ adenine aqueous solution to form drops with a diameter of $\sim 3 \mathrm{~mm}$ at the $2 \mathrm{D} \mathrm{Al}$ nanocrystal film/Si boundary, the $2 \mathrm{D} \mathrm{Al}$ nanocrystal film, and the $\mathrm{Si}$ substrate, respectively. Raman maps of $50 \mu \mathrm{m} \times 50 \mu \mathrm{m}$ area were collected with a $0.5 \mu \mathrm{m}$ step 

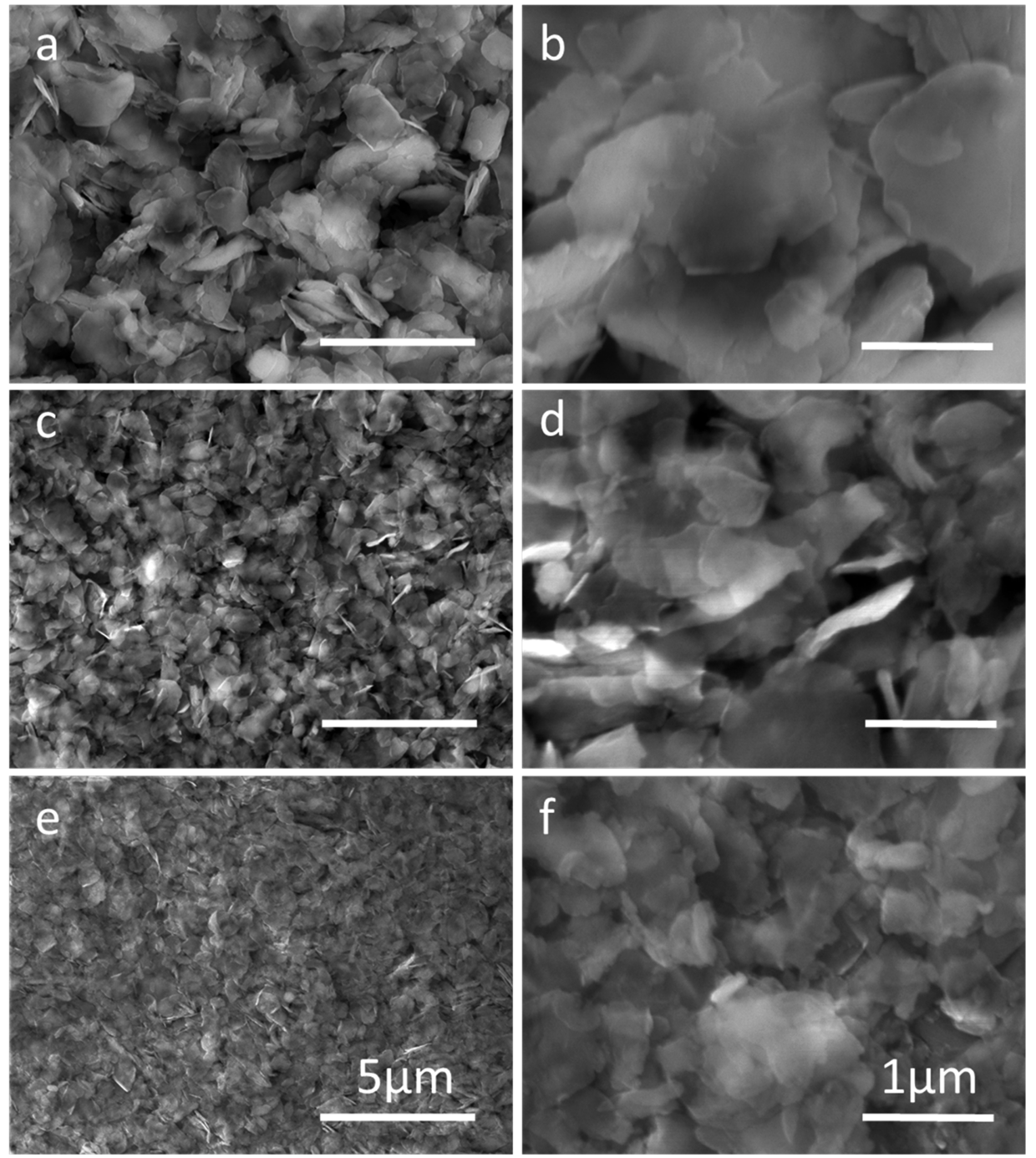

Figure 2. SEM images of 2D Al nanocrystals centrifugated at different speeds: (a and b) centrifugated at 10000 rpm; (c and d) centrifugated at $15000 \mathrm{rpm}$; (e and f) centrifugated at $20000 \mathrm{rpm}$. The scale bars in the left panels are $5 \mu \mathrm{m}$, and those in the right panels are $1 \mu \mathrm{m}$.

size with $1 \mathrm{~s}$ accumulation time after the adenine drops dried in a hood at the room temperature.

\section{RESULTS AND DISCUSSION}

The morphology of the Al nanoflakes separated with different centrifugation speeds were analyzed using SEM images, which showed that $\mathrm{Al}$ foils were transformed into $2 \mathrm{D} \mathrm{Al}$ nanocrystals of various sizes with ultrasonication (Figure 2a-f). The majority of the $2 \mathrm{D} \mathrm{Al}$ nanocrystals from $10000 \mathrm{rpm}$ separation were about $1-2 \mu \mathrm{m}$ (Figure $2 \mathrm{a}, \mathrm{b}$ ) in size and around $80-100$ $\mathrm{nm}$ in thickness. The thickness was obtained from the $2 \mathrm{D}$ crystals that were "standing up". The nanocrystals obtained from $15000 \mathrm{rpm}$ separation (Figures $2 \mathrm{c}$,d) were about $1 \mu \mathrm{m}$ in size with the thickness of less than $\sim 50 \mathrm{~nm}$. $20000 \mathrm{rpm}$ separation yields $\mathrm{Al}$ nanoflakes that are mostly smaller than $\sim 500 \mathrm{~nm}$ in size and about $20-30 \mathrm{~nm}$ in thickness (Figure $2 \mathrm{e}, \mathrm{f})$.

XRD patterns (Figure 3a) of the $2 \mathrm{D} \mathrm{Al}$ nanocrystals centrifugated at different speeds show four characteristic peaks for FCC phase aluminum (JCPDS \# 04-0787), corresponding to Miller indices (111), (200), (220), and (311). A representative low-resolution TEM image of $2 \mathrm{D} \mathrm{Al}$ nanocrystals (Figure 3b) shows that ultrasonically generated 2D Al nanocrystals have a relatively large distribution in particle size and particle thickness without size selection. A low-resolution TEM image (Figure 3c) of 2D Al nanocrystals separated at $15000 \mathrm{rpm}$ shows more uniform 2D Al nanocrystals after the "size selection" with some nanoflakes "laying down" flat and some "standing up" on the TEM grid. The dark "standing up" nanocrystals show that the thickness of the crystals is about 20-50 nm which agrees with what was estimated from the SEM images (Figure 2c,d). The high-resolution TEM image (Figure 3d) shows that the outer edges of the $\mathrm{Al}$ crystal were surrounded by a $7 \mathrm{~nm}$ amorphous $\mathrm{Al}_{2} \mathrm{O}_{3}$ layer. The measured interplanar spacing of the inside crystal is $0.236 \mathrm{~nm}$ (Figure 3d) which corresponds to the distance between $\mathrm{Al}$ (111) lattice planes. Both the SEM and TEM investigations clearly show that the $\mathrm{Al}$ foil was ultrasonically exfoliated into $2 \mathrm{D} \mathrm{Al}$ nanocrystals. The impervious $\mathrm{Al}_{2} \mathrm{O}_{3}$ layer prevents the $2 \mathrm{D} \mathrm{Al}$ nanocrystals from further oxidation (as in the $\mathrm{Al}$ foil) ${ }^{4,35,36}$ and makes them stable in ambient environment.

In order to understand the mechanism of the ultrasonic exfoliation of $\mathrm{Al}$ foil into $2 \mathrm{D}$ nanocrystals, we investigated the morphological and structural changes of the $\mathrm{Al}$ foil before and during ultrasonication using various microscopies. Figure $4 \mathrm{a}$ is a representative SEM image of $\mathrm{Al}$ foil used in our investigation 

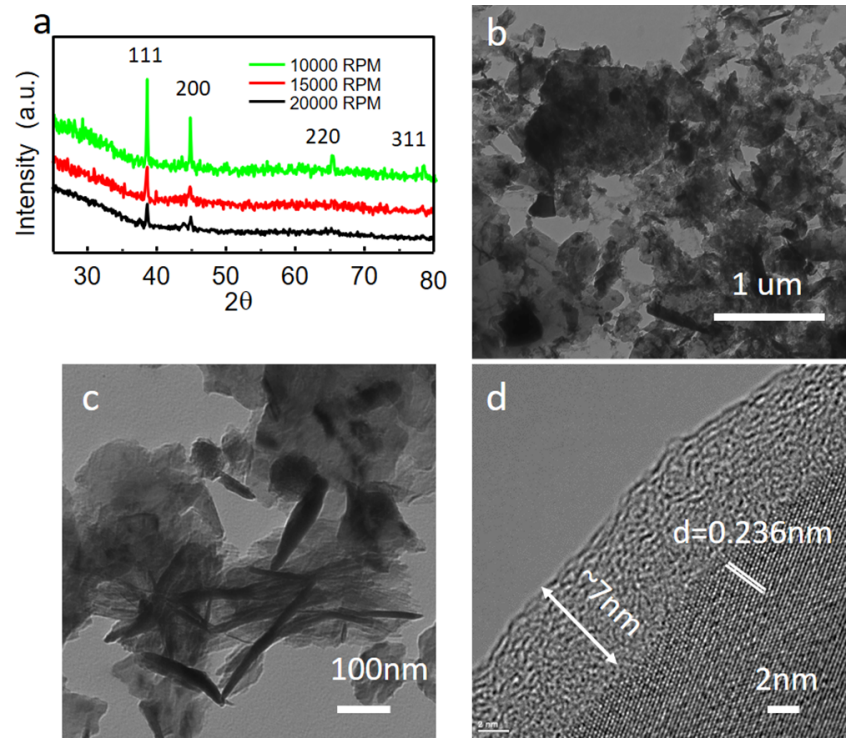

Figure 3. Structural analysis of $2 \mathrm{D} \mathrm{Al}$ nanocrystals. (a) XRD patterns of different-sized 2D Al nanocrystals; (b) low-resolution TEM image of $\mathrm{Al}$ nanocrystals without size selection; (c) low resolution TEM image of 2D Al nanocrystals separated at $15000 \mathrm{rpm}$; (d) high resolution TEM image of an $\mathrm{Al}$ nanocrystal covered with an amorphous $\mathrm{Al}_{2} \mathrm{O}_{3}$ layer.

before ultrasonication. The grooves and ridges are parallel to the rolling direction of the $\mathrm{Al}$ foil during manufacturing. The distance between the grooves or ridges obtained from SEM images (Figure $4 \mathrm{a}$ and Figure S2a) is about $10-40 \mu \mathrm{m}$. Aluminum foil is produced by rolling $\mathrm{Al}$ ingots into the desired thickness through continuous casting or cold rolling. The grains in the $\mathrm{Al}$ sheet elongate in the rolling direction and the thickness is decreased in the normal direction. The Al foil product consists of layers of highly elongated, deformed grains with the desired thickness. ${ }^{37}$ The microsteps induced by the grains overlapping at their boundaries due to grain boundary sliding are highlighted with white circles in Figure $4 b .^{38,39}$ The AFM image (Figure 4c) shows that there are shallow parallel grooves and ridges inside the grooves observed in the SEM images (Figure 4a and Figure S2a). These shallow grooves and ridges are caused by the multiple rolling process. The inserted AFM line profile (Figure 4c) shows that the depth of the shallow grooves and ridges is about 10 to $30 \mathrm{~nm}$, which is much smoother than the structure from last roller because each rolling pass slightly reduces the surface roughness of $\mathrm{Al}$ surface. ${ }^{40,41}$ The dents with depth of about $80 \mathrm{~nm}$ caused by a roller or post rolling process ${ }^{38,42}$ can also be identified in the AFM image (Figure S2b,c).

In general, the initial ultrasonic process yields a wrinkled $\mathrm{Al}$ foil with perforated holes. The Al foil broke into small pieces when the perforated holes interconnected. Figure $4 \mathrm{~d}$ shows the SEM image of Al foil after $30 \mathrm{~min}$ of sonication in ethylene glycol. Damages with the size around $80-180 \mu \mathrm{m}$ are randomly located on the sonicated Al foil surface. Some of the damaged areas were perforated into holes, and some areas formed dents (highlighted in circles) without through penetration. $^{27}$ Figure $4 \mathrm{e}$ presents a high-resolution SEM image of the edge area of a perforated hole highlighted with a square in Figure 4d, which shows a clear lamellated structure. Besides the addition of perforated holes, the surface grooves and ridges from the rolling process gradually diminished with longer ultrasonication (Figure S3a). The zoomed-in SEM image (Figure S3b) clearly reveals cleavage steps and parallel
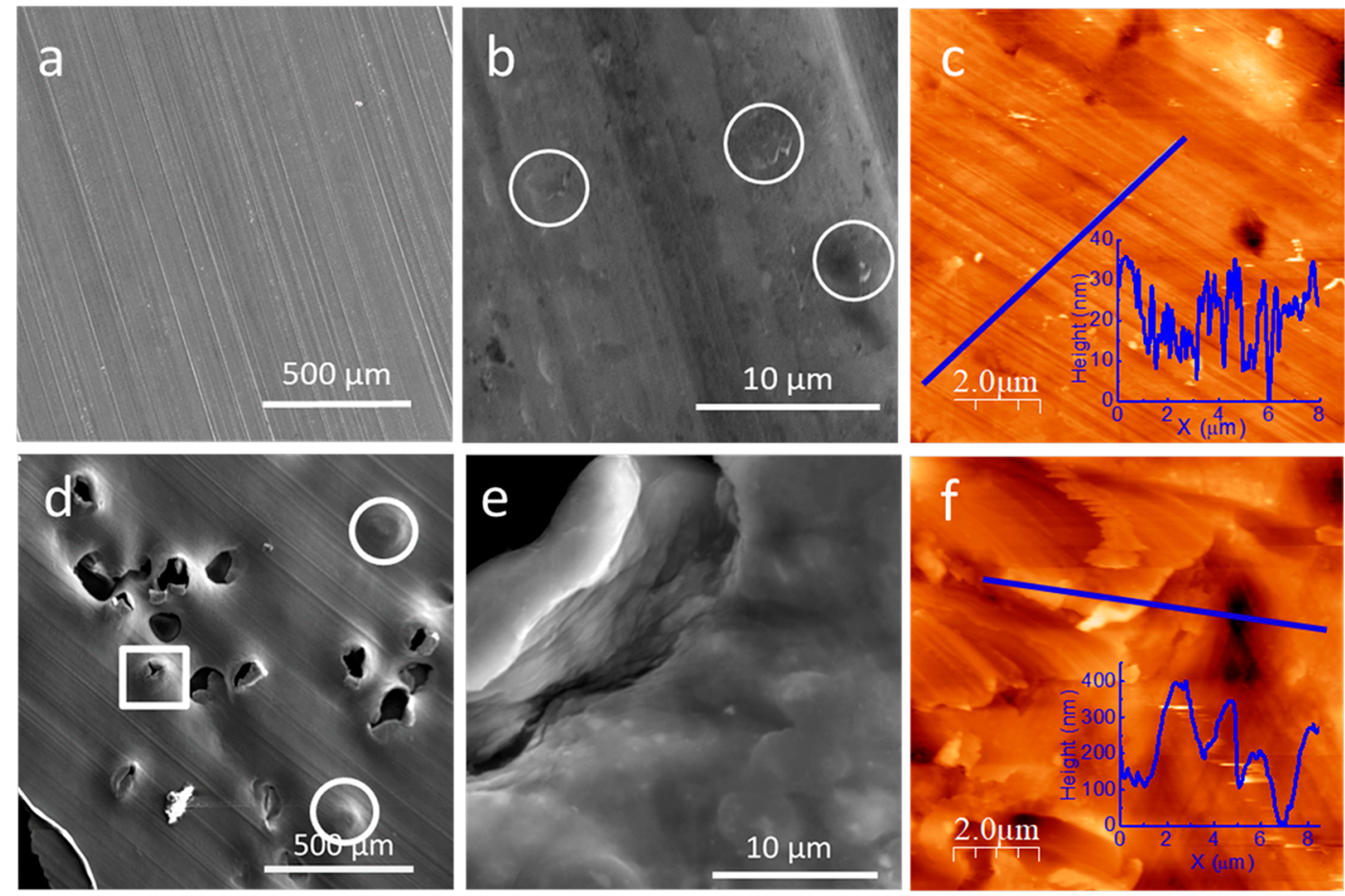

Figure 4. Structural analysis of Al foil before and during ultrasonication. (a) Representative SEM image of Al foil; (b) high magnification SEM image of Al foil shows grain boundaries (highlighted with white circles); (c) AFM image and line profile of Al foil surface; (d) SEM image of sonicated $\mathrm{Al}$ foil shows perforated holes (highlighted in square) and dents (highlighted in circles); (e) SEM image of vicinity area of a perforated hole highlighted in panel d; (f) AFM image and line profile of vicinity area of a perforated hole on sonicated Al foil. 

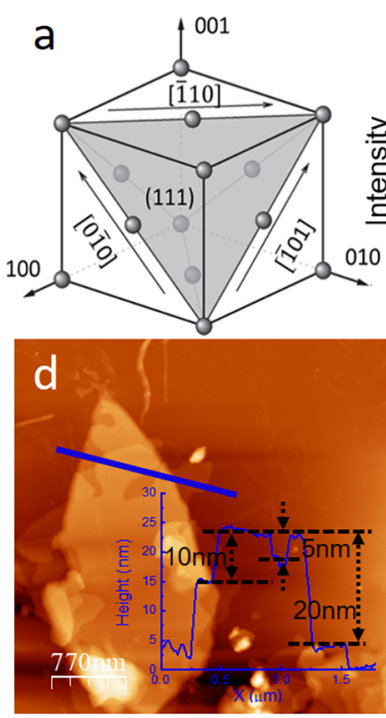

b
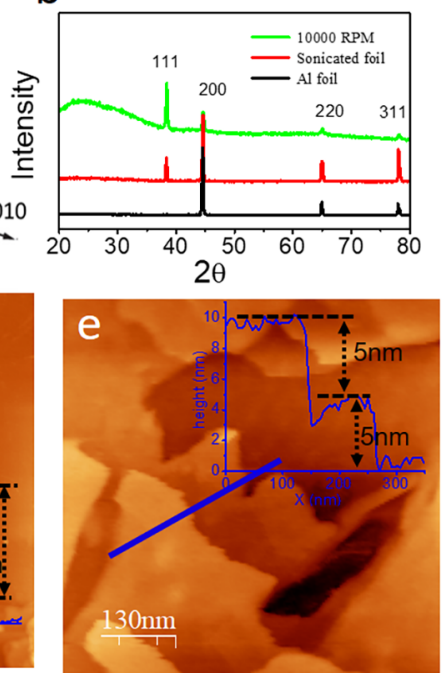

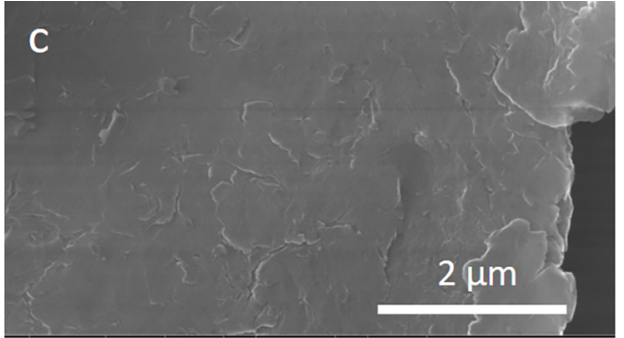

f

g

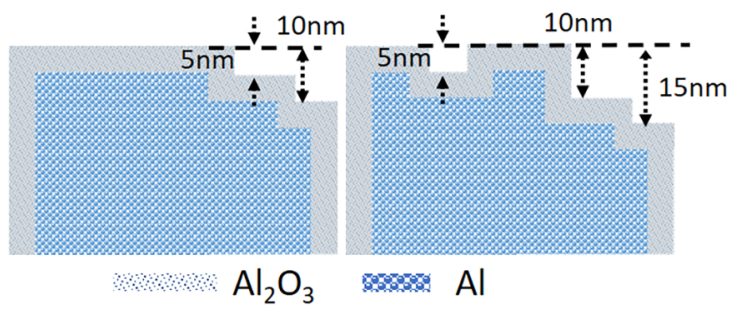

Figure 5. Structural analysis and exfoliation mechanism of 2D Al nanocrystals. (a) Octahedral slip systems in FCC crystal (reproduced from ref 45) ; (b) XRD patterns of Al foil (black), sonicated $\mathrm{Al}$ foil (red) and 2D Al nanocrystals (green); (c) SEM image shows the flaked structures on a 2D Al crystal surface; (d) AFM image shows step thicknesses on the surface of a $2 \mathrm{D}$ Al crystal; (e) AFM image shows features due to layer by layer exfoliation (inserted line profile shows the thickness of $\mathrm{Al}_{2} \mathrm{O}_{3}$ layer is $5 \mathrm{~nm}$ ); ( $\mathrm{f}$ and $\mathrm{g}$ ) sketch of layer-by-layer exfoliation mechanism.

facets after the surface grooves and ridges were removed by sonication. Figure $4 \mathrm{f}$ presents an AFM image of the edge area of a perforated hole. Lamellar structures of 200-300 nm thickness (inserted line profile in Figure 4f) are similar to the exfoliated 2D Mg nanocrystals. ${ }^{25}$ As mentioned above, there are abundant slip planes in Al. Slip is the dominant deformation model ${ }^{43-45}$ when the $\mathrm{Al}$ sheet is rolled into $\mathrm{Al}$ foil with massive thickness reduction resulting in layers of highly elongated, deformed grains shown as lamellar structures in the SEM (Figure 4b) and AFM (Figure 4f) images. Lamellar structures with 200-300 nm thickness fluctuation (inserted line profile in Figure 4f) caused by metals deform inhomogeneously through thickness reduction due to a large amount of shear strain, ${ }^{46}$ which is also observed in exfoliated 2D Mg nanocrystals. ${ }^{25}$ The surface areas on sonicated foil that is far from perforated holes show similar surface roughness as that of the nonsonicated surface (Figure S4).

The textural structures of the $\mathrm{Al}$ foil are determined by the crystal structure of $\mathrm{Al}$ and its manufacturing process. $\mathrm{Al}$ has an FCC structure (Figure 5a) with relatively high stacking-fault energy (SFE) mainly deformed by $\{111\}<110>$ slip. $^{43,47,48}$ When a heavy cold-rolled aluminum specimen is annealed under heat treatment conditions, one of the origins of $\{100\}\langle 001\rangle$ recrystallization nuclei is considered to be the $\{100\}<001>$ band existing in the deformed structure, called "the cube band". 99,50 Figure $5 \mathrm{~b}$ compares the XRD patterns of $\mathrm{Al}$ foil, sonicated foil, and $\mathrm{Al} 2 \mathrm{D}$ nanocrystals. The strongest peak in XRD pattern of the $\mathrm{Al}$ foil (black line in Figure $4 \mathrm{~b}$ ) at (200) $\left(2 \theta=44.6^{\circ}\right)$ and negligible $(111)$ signal confirms that the surface of the $\mathrm{Al}$ foil is mainly $\{100\}<001>$ as in the "cube band" textures with the $\{100\}$ plane parallel to the rolling plane and the $\langle 100\rangle$ direction along the rolling direction. ${ }^{51}$ Comparison of the XRD pattern of ultrasonicated $\mathrm{Al}$ foil with that of $\mathrm{Al}$ foil shows that one significant change is the appearance of the (111) peak, which suggests that a substantial amount of additional $\{111\}$ planes were exposed after the surface "cube band" textures were exfoliated. The surface energy of $\mathrm{Al}$ low index planes has the order of $(111)<(100)<$
(110)..$^{52,53}$ Therefore, it is not surprising that the preferred cleavage plane of the FCC Al lattice by ultrasonication is determined to be (111) as confirmed by both XRD results and high-resolution TEM observation (Figure $3 \mathrm{~d}$ ). In addition to the (111) peak, there is a broad peak (centered at $2 \theta=25^{\circ}$ ) in the XRD pattern of $2 \mathrm{D} \mathrm{Al}$ nanocrystals; this broad peak can be assigned to amorphous $\mathrm{Al}_{2} \mathrm{O}_{3} \cdot{ }^{54}$ Further SEM measurements on the broken pieces of the $2 \mathrm{D} \mathrm{Al}$ microcrystals provide important morphological information to understand the exfoliation process.

The SEM image (Figure 5c) of an Al microcrystal from 2000 rpm reveals that there are many nanoflakes on the surface of the $\mathrm{Al}$ microcrystal. Additional SEM images confirm that most of the microsized $\mathrm{Al}$ crystals have similar flaked surface features (Figures S5). Figure 5d presents an AFM image of an $\mathrm{Al}$ microcrystal. The line profile shows that the height of the flaked surface features is roughly in multiples of $5 \mathrm{~nm}$. Figure $5 \mathrm{e}$ is an AFM image taken on a representative $2 \mathrm{D} \mathrm{Al}$ microcrystal with flaked step structures. The most surprising finding is that most of the steps have an almost identical height of $5 \mathrm{~nm}$ or multiples of $5 \mathrm{~nm}$. Since the surface of an $\mathrm{Al}$ metal is always covered with an $\mathrm{Al}_{2} \mathrm{O}_{3}$ layer of a few nanometers in ambient environment, ${ }^{4,35,36}$ it is reasonable to conclude that the $5 \mathrm{~nm}$ flake structures are a layer of $\mathrm{Al}_{2} \mathrm{O}_{3}$ with the most of their thickness of $\sim 5 \mathrm{~nm}$ (Figure 5f). The thickness difference between the AFM and TEM observations can be attributed to the differences in the resolution of AFM and TEM techniques. The apparent height obtained from AFM measurements may be affected by sample surface conditions, tip-samples interaction, vibration, and instrumental offset. ${ }^{55-57}$

On the basis of these microscopy observations, we propose that when $\mathrm{Al}$ foil breaks into small pieces or is exfoliated into $2 \mathrm{D}$ nanocrystals, the process is assisted via the $\mathrm{Al}_{2} \mathrm{O}_{3}$ layer. The $5 \mathrm{~nm} \mathrm{Al}_{2} \mathrm{O}_{3}$ layer can be exfoliated under continuous ultrasonication, and then the newly exposed $\mathrm{Al}$ surface is oxidized to $\mathrm{Al}_{2} \mathrm{O}_{3}$ again (Figure $5 \mathrm{~g}$ ). By repeating this $\mathrm{Al}_{2} \mathrm{O}_{3}$ exfoliation process, a layer-by-layer exfoliation of $\mathrm{Al}_{2} \mathrm{O}_{3}$ can lead to the production of thinner $2 \mathrm{D} \mathrm{Al}$ nanocrystals. On one 

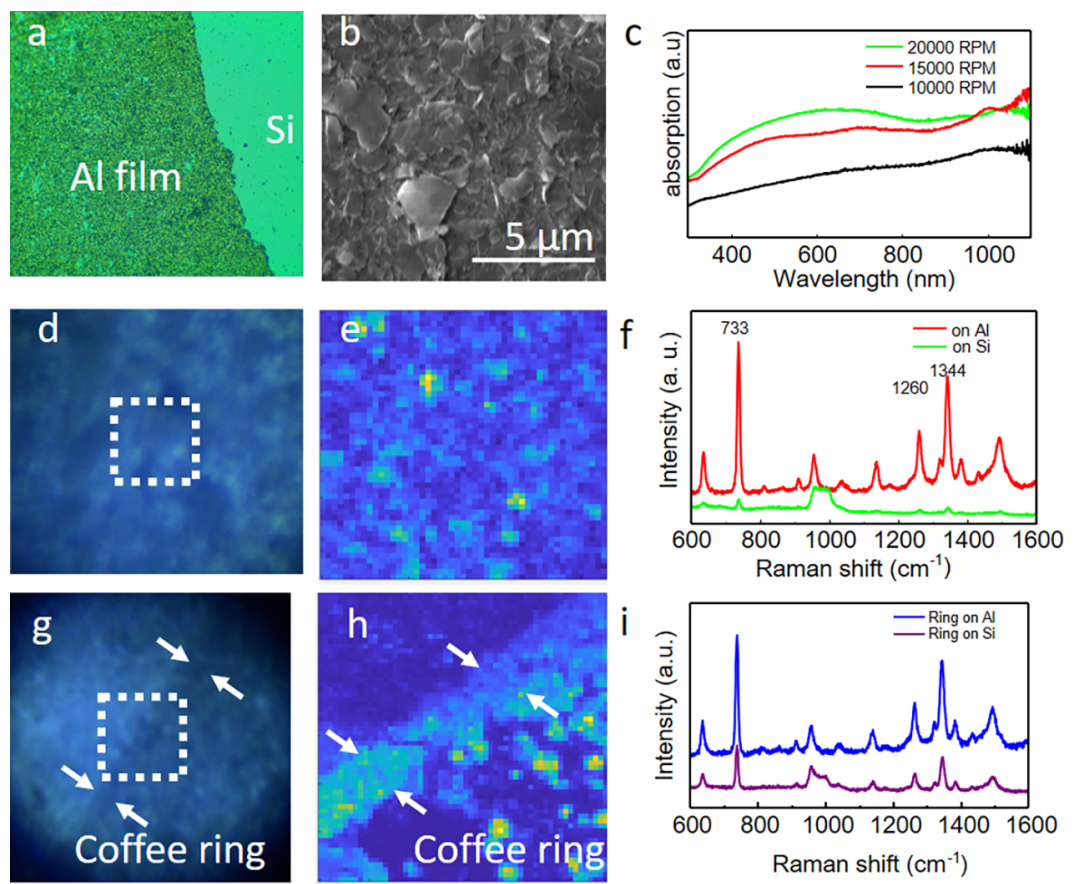

Figure 6. SERS of adenine on self-assembled $2 \mathrm{D} \mathrm{Al}$ nanocrystal film. Optical image (a) and SEM image (b) of $2 \mathrm{D}$ Al nanocrystal films on Si substrate; (c) UV-vis absorption spectra of thin films self-assembled 2D Al nanocrystals separated at different centrifugation speeds; (d) optical image of adenine on $\mathrm{Al} \mathrm{film} \mathrm{inside} \mathrm{the} \mathrm{"coffee} \mathrm{ring";} \mathrm{(e)} \mathrm{Raman} \mathrm{mapping} \mathrm{of} \mathrm{adenine} \mathrm{from} \mathrm{the} \mathrm{area} \mathrm{highlighted} \mathrm{in} \mathrm{panel} \mathrm{d;} \mathrm{(f)} \mathrm{averaged} \mathrm{Raman}$ spectra of adenine inside the "coffee ring" on $\mathrm{Al}$ film and on $\mathrm{Si}$; $\mathrm{g}$ ) optical image of adenine "coffee ring" on Al film highlighted by arrows; (h) Raman mapping of adenine from the area highlighted in panel g; (i) averaged Raman spectra of adenine "coffee ring" on Al film and on Si.

hand, the surface $\mathrm{Al}_{2} \mathrm{O}_{3}$ layer protects the $2 \mathrm{D} \mathrm{Al}$ nanocrystals from further oxidization; on the other hand, the surface $\mathrm{Al}_{2} \mathrm{O}_{3}$ layer can be further exfoliated to reduce the thickness of $2 \mathrm{D} \mathrm{Al}$ nanocrystals.

The 2D Al nanocrystals assembled into Al nanocrystal films on the water/air interface when the $\mathrm{Al}$-ethanol mixture was carefully drop-cast onto a water surface as the ethanol evaporated (Figure 1d). The floating white film of $2 \mathrm{D} \mathrm{Al}$ nanocrystals can be transferred to different substrates (inset of Figure 1d). The optical image shows that $2 \mathrm{D} \mathrm{Al}$ nanocrystals form a relatively uniform film (Figure $6 \mathrm{a}$ and Figure S7a) on substrates. The SEM image (Figure $6 \mathrm{~b}$ and Figure S7b) of the 2D Al nanocrystal film shows that most of the $2 \mathrm{D} \mathrm{Al}$ nanocrystals "lay" flat on Si substrate. This is in contrast to the randomly orientated $2 \mathrm{D} \mathrm{Al}$ nanocrystals in films prepared by directly drop-casting the $\mathrm{Al}$-ethanol mixture on $\mathrm{Si}$ substrates (Figure S7c,d).

The optical absorption of $2 \mathrm{D} \mathrm{Al}$ nanocrystal films on quartz substrates was investigated with a UV-vis spectrometer (Agilent 8453). No clear plasmonic resonance peaks were observed in any of the 2D Al nanocrystal thin films. Broadband absorption across the entire visible range ( 300 to $800 \mathrm{~nm}$ ) was observed from thin films made of $\mathrm{Al}$ nanocrystals separated at 15000 and $20000 \mathrm{rpm}$ (Figure 6c) because the size of the $\mathrm{Al}$ nanocrystals centrifugated at higher centrifugation speeds is smaller than that of the $\mathrm{Al}$ nanocrystals collected at lower speed. The stronger absorption from smaller crystals is consistent with what is expected from the plasmonic resonance of $\mathrm{Al}$ nanocrystals. The experiments and simulations of uniform $\mathrm{Al}$ nanodiscks prepared via electron-beam evaporation show that only smaller $\mathrm{Al}$ nanodisks exhibit the characteristic Lorentzian resonance of a dipolar oscillator. As nanodisk diameter is increased, the plasmon resonance red shifts and broadens and introduces higher-order, multipolar resonances. ${ }^{4}$ Similar broad but weaker absorption spectra were observed when the $\mathrm{Al} 2 \mathrm{D}$ nanocrystals were dispersed in ethanol solution (Figure S6). We attribute the broadband absorption to the broad size distribution and the random shape of the particles. $^{25,55}$ The relatively large size of the $\mathrm{Al}$ nanocrystals could also contribute to the broadband absorption due to higher-order, multipolar resonances. ${ }^{56,57}$

To measure the surface enhancement of the Raman signal of the DNA base adenine on self-assembled 2D Al nanocrystal film, a drop of $3 \mu \mathrm{L}$ of adenine $10^{-4} \mathrm{M}$ solution was pipetted at the distinct $\mathrm{Al}-\mathrm{Si}$ substrate boundary (Figure 6a). Raman spectra were obtained from both the Si substrate and the $2 \mathrm{D} \mathrm{Al}$ nanocrystal film from the same droplet of adenine solution. With the evaporation of the solvent, a coffee-ring like pattern remained on both $\mathrm{Al}$ film and $\mathrm{Si}$ substrate. The periphery of the "coffee ring" was covered with heterogeneous adenine caused by the accumulation of molecules at the drying boundaries, while the inside area of the "coffee ring" was covered with a relatively uniform layer of adenine. ${ }^{58-61}$ Raman spectra were taken inside "coffee ring" and on the "coffee ring" on both 2D Al film and Si. Figures 6d,e are the optical image and the Raman map of adenine taken inside the "coffee ring" on $\mathrm{Al}$ film, respectively. The Raman intensity map (Figure 6e) of the $733 \mathrm{~cm}^{-1}$ peak shows that adenine is distributed evenly across the scanned area, confirming a relatively uniform layer of adenine inside the "coffee ring" (although there are a few "hot spots" with stronger adenine signals). Figure $6 f$ shows the average Raman spectra of adenine inside the "coffee ring" on $\mathrm{Si}$ and 2D Al nanocrytal film. Only the strongest Raman shifts at 733,1260 , and $1344 \mathrm{~cm}^{-1}$ can be identified on the averaged spectrum (green line) from the $\mathrm{Si}$ area. The broad peak between 950 and $1020 \mathrm{~cm}^{-1}$ is from the secondary scattering 
of the Si substrate. All the characteristic Raman peaks (red line) of adenine molecules are clearly visible from $2 \mathrm{D} \mathrm{Al}$ nanocrystal film and are much stronger than those obtained from $\mathrm{Si}$ area, indicating that the $2 \mathrm{D} \mathrm{Al}$ film surface enhances the Raman scattering of adenine molecules inside the adenine "coffee ring". 62

Analytical enhancement factor (AEF) was proposed by Le $\mathrm{Ru}$ et al. ${ }^{63}$ and is well accepted for practical applications under identical experimental conditions. ${ }^{63-66}$ This factor addresses the apparent enhancement from SERS compared to spontaneous Raman scattering when the Raman signals are not only from the absorbed surface but also from the distant area. An AEF of 18 is calculated inside the ring which is the peak area ratio of $733 \mathrm{~cm}^{-1}$ Raman shift of adenine on $\mathrm{Al}\left(I_{\mathrm{Al}}\right)$ to that on $\mathrm{Si}\left(I_{\mathrm{Si}}\right)$. As for the "coffee ring" area, the AEF is about 11 (Figure 6i). On the optical image of the "coffee ring" area, the interface of "with adenine" and "without adenine" on the $\mathrm{Al}$ film is clearly distinct across the diagonal of the picture as indicated by the white arrows (Figure 6g). From the Raman mapping (Figure $6 \mathrm{~h}$ ), it is clear that adenine molecules are distributed heterogeneously on the "coffee ring". The AEF value on the "coffee ring" is less than the value inside the coffee ring because the aggregation of the adenine molecules results in relatively fewer adenine molecules that are in the SERS scattering volume and are directly interacting with the $\mathrm{Al} 2 \mathrm{D}$ film substrate. To eliminate the possibility that more adenine molecules were accumulated on $\mathrm{Al}$ film when the adenine solution was dropped at the $2 \mathrm{D} \mathrm{Al}$ nanocrystal film/Si boundary, Raman measurements of single droplets of $10^{-4} \mathrm{M}$ adenine solution drop-casted on $\mathrm{Si}$ substrate and $2 \mathrm{D} \mathrm{Al}$ nanocrystal film separately were carried out (Figure S8a,b). The AEF values obtained on the coffee ring and inside the coffee ring are 13 and 19 , respectively, which are close to the values obtained from the same adenine droplet at the $2 \mathrm{D} \mathrm{Al}$ nanocrystal film/Si boundary. To determine the detection limit of adenine on 2D Al nanocrystals film, Raman signals of adenine solutions of various concentrations were measured (Figure S8c). The detection limit was determined to be $\sim 10^{-7}$ M.

Up to this point, we have been referring to enhancements in terms of AEF, which represents increased signal intensity but does not account for factor of the number of molecules contributing to SERS signal. Since the enhancement of SERS is due to the localized surface plasmons, only the molecules adsorbed within $\sim 10 \mathrm{~nm}$ from the nanoparticles' surface contribute to SERS. ${ }^{66}$ To account for this factor and to gain a deeper understanding of the mechanisms, we calculated the more commonly used average SERS enhancement factor (EF $E_{\text {SERS }}$ ) (S9). The $\mathrm{EF}_{\text {SERS }}$ of adenine on our $\mathrm{Al} 2 \mathrm{D}$ nanocrystal film is $\sim 4 \times 10^{2}$. Although this enhancement is quite weak compared with $\mathrm{Au}$ and silver substrates, it is comparable to the reported SERS enhancement factor $\left(\mathrm{EF}_{\mathrm{SERS}}\right.$ $=1 \times 10^{3}$ ) of adenine on aluminum film-over nanosphere substrates for UV surface-enhanced Raman scattering with a $229 \mathrm{~nm}$ excitation. ${ }^{67}$

Besides the electromagnetic effect, chemical enhancement occurs when charge transfer takes place between the molecules and substrate. It has been shown that the alumina nanotubes/ nanopores without plasmonic metal deposits are SERS inactive. ${ }^{68}$ In fact, the atomic layer deposited thin $\mathrm{Al}_{2} \mathrm{O}_{3}$ layer was used to eliminate the chemical enhancement by blocking charge transfer between the molecules and the metal surface. ${ }^{69,70}$ Therefore, we conclude that the SERS observed in the experiments are from aluminum plasmonic resonance.

As expected, although clear Raman enhancements of adenine were observed with $2 \mathrm{D}$ Al nanocrystal film using a $532 \mathrm{~nm}$ laser excitation, the enhancement is much smaller than what is usually observed on $\mathrm{Au}$ and $\mathrm{Ag}$ SERS substrates. Previous simulations showed that the optimal enhancement by $\mathrm{Al}$ with a native $\mathrm{Al}_{2} \mathrm{O}_{3}$ coating is about 2 orders of magnitude less than that for $\mathrm{Al}^{71}$ However, there is still a substantial increase in Raman signals observed experimentally. ${ }^{67,71} \mathrm{Al}$ nanostructures have shown a great potential for plasmonic applications in DUV -NIR even with the $\mathrm{Al}_{2} \mathrm{O}_{3}$ layers. ${ }^{67,71-76}$

\section{CONCLUSIONS}

In summary, we developed a simple and economical but effective synthetic method to generate $2 \mathrm{D} \mathrm{Al}$ nanocrystals from commercially available $\mathrm{Al}$ foil. The external stress from ultrasonic cavitation enables preferred cleavage along the (111) surface planes and the exfoliation of deformed $\mathrm{Al}$ grains from the $\mathrm{Al}$ foil into $2 \mathrm{D} \mathrm{Al}$ nanocrystals. The $2 \mathrm{D} \mathrm{Al}$ nanocrystals of different sizes can be separated by centrifugation. Layer-by-layer $\mathrm{Al}_{2} \mathrm{O}_{3}$ exfoliation from the surface of the $2 \mathrm{D} \mathrm{Al}$ nanocrystals leads to thinner $2 \mathrm{D} \mathrm{Al}$ nanocrystals. The 2D Al nanocrystals self-assemble into an $\mathrm{Al}$ nanocrystal thin film at the water/air interface which can be transferred to different substrates. The self-assembled $2 \mathrm{D} \mathrm{Al}$ nanocrystal thin films show SERS effect in adenine Raman measurement using visible laser excitation. It will be worthwhile to explore the ultrasonic exfoliation method for the production of $2 \mathrm{D}$ metallic nanocrystals from other metallic foils.

\section{ASSOCIATED CONTENT}

\section{Supporting Information}

The Supporting Information is available free of charge at https://pubs.acs.org/doi/10.1021/acs.jpcc.0c10935.

(PDF)

\section{AUTHOR INFORMATION}

\section{Corresponding Authors}

Weigang Lu - Department of Physics and Department of Chemistry and Biochemistry, Baylor University, Waco, Texas 76798, United States; 이이.org/0000-0002-9016-2729; Email: Weigang_Lu@baylor.edu

Zhenrong Zhang - Department of Physics, Baylor University, Waco, Texas 76798, United States; ○orcid.org/00000003-3969-2326; Email: Zhenrong_zhang@baylor.edu

Touradj Solouki - Department of Chemistry and Biochemistry, Baylor University, Waco, Texas 76798, United States; 아이.org/0000-0002-4209-9200;

Email: Touradj_Solouki@baylor.edu

\section{Authors}

Blake Birmingham - Department of Physics, Baylor University, Waco, Texas 76798, United States

Dmitri V. Voronine - Department of Physics and Department of Medical Engineering, University of South Florida, Tampa, Florida 33620, United States; Oorcid.org/0000-00028841-7657

Drew Stolpman - Department of Chemistry and Biochemistry, Baylor University, Waco, Texas 76798, United States 
Sharad Ambardar - Department of Medical Engineering, University of South Florida, Tampa, Florida 33620, United States

Deniz Altunoz Erdogan - Department of Chemistry, Bilkent University, 06800 Bilkent, Ankara, Turkey

Emrah Ozensoy - Department of Chemistry, Bilkent University, 06800 Bilkent, Ankara, Turkey; (1) orcid.org/ 0000-0003-4352-3824

Complete contact information is available at: https://pubs.acs.org/10.1021/acs.jpcc.0c10935

\section{Notes}

The authors declare no competing financial interest.

\section{ACKNOWLEDGMENTS}

Acknowledgment is made to the Donors of the American Chemical Society Petroleum Research Fund for partial support of this research. This work was partially supported by the National Science Foundation under Grant CHE-1905043 and the Office of vice Provost for Research of Baylor University. The authors would like to thank Dr. Bernd Zechmann (Center for Microscopy and Imaging, Baylor University, Waco, TX) for technical support in microscopy measurements and image analysis.

\section{REFERENCES}

(1) Murray, W. A.; Suckling, J. R.; Barnes, W. L. Overlayers on Silver Nanotriangles: Field Confinement and Spectral Position of Localized Surface Plasmon Resonances. Nano Lett. 2006, 6, 1772-1777.

(2) Nehl, C.; Liao, H.; Hafner, J. Optical Properties of Star-Shaped Gold Nanoparticles. Nano Lett. 2006, 6, 683-688.

(3) Hornyak, G.; Phani, K.; Kunkel, D.; Menon, V.; Martin, C. Fabrication, Characterization and Optical Theory of Aluminum Nanometal Nanoporous Membrane Thin Film Composites. Nanostruct. Mater. 1995, 6, 839-842.

(4) Knight, M. W.; King, N. S.; Liu, L.; Everitt, H. O.; Nordlander, P.; Halas, N. J. Aluminum for Plasmonics. ACS Nano 2014, 8, 834840.

(5) Ambardar, S.; Nguyen, D.; Binder, G.; Withers, Z. W.; Voronine, D. V. Quantum Leap from Gold and Silver to Aluminum Nanoplasmonics for Enhanced Biomedical Applications. Appl. Sci. 2020, 10, 4210.

(6) Mogensen, K. B.; Guhlke, M.; Kneipp, J.; Kadkhodazadeh, S.; Wagner, J. B.; Espina Palanco, M.; Kneipp, H.; Kneipp, K. SurfaceEnhanced Raman Scattering on Aluminum using Near Infrared and Visible Excitation. Chem. Commun. 2014, 50, 3744-3746.

(7) Martin, J.; Proust, J.; Gerard, D.; Plain, J. Localized Surface Plasmon Resonances in the Ultraviolet from Large Scale Nanostructured Aluminum Films. Opt. Mater. Express 2013, 3, 954-959.

(8) Langhammer, C.; Schwind, M.; Kasemo, B.; Zoric, I. Localized Surface Plasmon Resonances in Aluminum Nanodisks. Nano Lett. 2008, 8, 1461-1471.

(9) Chowdhury, M. H.; Ray, K.; Gray, S. K.; Pond, J.; Lakowicz, J. R. Aluminum Nanoparticles as Substrates for Metal-Enhanced Fluorescence in the Ultraviolet for the Label-Free Detection of Biomolecules. Anal. Chem. 2009, 81, 1397-1403.

(10) Jha, S. K.; Ahmed, Z.; Agio, M.; Ekinci, Y.; Loeffler, J. F. DeepUV Surface-Enhanced Resonance Raman Scattering of Adenine on Aluminum Nanoparticle Arrays. J. Am. Chem. Soc. 2012, 134, 19661969.

(11) Maidecchi, G.; Gonella, G.; Zaccaria, R. P.; Moroni, R.; Anghinolfi, L.; Giglia, A.; Nannarone, S.; Mattera, L.; Dai, H.; Canepa, M.; Bisio, F. Deep Ultraviolet Plasmon Resonance in Aluminum Nanoparticle Arrays. ACS Nano 2013, 7, 5834-5841.

(12) Taguchi, A.; Saito, Y.; Watanabe, K.; Yijian, S.; Kawata, S. Tailoring Plasmon Resonances in the Deep-Ultraviolet by Size-
Tunable Fabrication of Aluminum Nanostructures. Appl. Phys. Lett. 2012, 101, 081110

(13) Canalejas-Tejero, V.; Herranz, S.; Bellingham, A.; MorenoBondi, M. C.; Barrios, C. A. Passivated Aluminum Nanohole Arrays for Label-Free Biosensing Applications. ACS Appl. Mater. Interfaces 2014, 6, 1005-1010.

(14) Sigle, D. O.; Perkins, E.; Baumberg, J. J.; Mahajan, S. Reproducible Deep-UV SERRS on Aluminum Nanovoids. J. Phys. Chem. Lett. 2013, 4, 1449-1452.

(15) Clark, B. D.; DeSantis, C. J.; Wu, G.; Renard, D.; McClain, M. J.; Bursi, L.; Tsai, A.; Nordlander, P.; Halas, N. J. Ligand-Dependent Colloidal Stability Controls the Growth of Aluminum Nanocrystals. J. Am. Chem. Soc. 2019, 141, 1716-1724.

(16) Clark, B. D.; Jacobson, C. R.; Lou, M.; Yang, J.; Zhou, L.; Gottheim, S.; DeSantis, C. J.; Nordlander, P.; Halas, N. J. Aluminum Nanorods. Nano Lett. 2018, 18, 1234-1240.

(17) Clark, B. D.; Jacobson, C. R.; Lou, M.; Renard, D.; Wu, G.; Bursi, L.; Ali, A. S.; Swearer, D. F.; Tsai, A.; Nordlander, P.; Halas, N. J. Aluminum Nanocubes have Sharp Corners. ACS Nano 2019, 13, 9682-9691.

(18) McClain, M. J.; Schlather, A. E.; Ringe, E.; King, N. S.; Liu, L.; Manjavacas, A.; Knight, M. W.; Kumar, I.; Whitmire, K. H.; Everitt, H. O.; Nordlander, P.; Halas, N. J. Aluminum Nanocrystals. Nano Lett. 2015, 15, 2751-2755.

(19) Lu, S.; Yu, H.; Gottheim, S.; Gao, H.; DeSantis, C. J.; Clark, B. D.; Yang, J.; Jacobson, C. R.; Lu, Z.; Nordlander, P.; Halas, N. J.; Liu, K. Polymer-Directed Growth of Plasmonic Aluminum Nanocrystals. J. Am. Chem. Soc. 2018, 140, 15412-15418.

(20) Robatjazi, H.; Weinberg, D.; Swearer, D. F.; Jacobson, C.; Zhang, M.; Tian, S.; Zhou, L.; Nordlander, P.; Halas, N. J. MetalOrganic Frameworks Tailor the Properties of Aluminum Nanocrystals. Science Advances 2019, 5, eaav5340.

(21) Swearer, D. F.; Robatjazi, H.; Martirez, J. M. P.; Zhang, M.; Zhou, L.; Carter, E. A.; Nordlander, P.; Halas, N. J. Plasmonic Photocatalysis of Nitrous Oxide into $\mathrm{N}_{2}$ and $\mathrm{O}_{2}$ using AluminumIridium Antenna-Reactor Nanoparticles. ACS Nano 2019, 13, 80768086.

(22) Jacobson, C. R.; Solti, D.; Renard, D.; Yuan, L.; Lou, M.; Halas, N. J. Shining Light on Aluminum Nanoparticle Synthesis. Acc. Chem. Res. 2020, 53, 2020-2030.

(23) Marrazzo, A.; Gibertini, M.; Campi, D.; Mounet, N.; Marzari, N. Prediction of a Large-Gap and Switchable Kane-Mele Quantum Spin Hall Insulator. Phys. Rev. Lett. 2018, 120, 117701.

(24) Mounet, N.; Gibertini, M.; Schwaller, P.; Campi, D.; Merkys, A.; Marrazzo, A.; Sohier, T.; Castelli, I. E.; Cepellotti, A.; Pizzi, G.; Marzari, N. Two-Dimensional Materials from High-Throughput Computational Exfoliation of Experimentally Known Compounds. Nat. Nanotechnol. 2018, 13, 246.

(25) Zhang, C.; Xu, Y.; Lu, P.; Wei, C.; Zhu, C.; Yao, H.; Xu, F.; Shi, J. Cryogenic Exfoliation of Non-Layered Magnesium into TwoDimensional Crystals. Angew. Chem., Int. Ed. 2019, 58, 8814-8818.

(26) Kusters, K. A.; Pratsinis, S. E.; Thoma, S. G.; Smith, D. M. Energy-Size Reduction Laws for Ultrasonic Fragmentation. Powder Technol. 1994, 80, 253-263.

(27) Santos, H. M.; Lodeiro, C.; Capelo-Martínez, J. In The Power of Ultrasound; Ultrasound in Chemistry; Wiley-VCH Verlag GmbH \& Co. KGaA: 2008; pp 1-16.

(28) Suslick, K. S.; Hammerton, D. A.; Cline, R. E. The Sonochemical Hot-Spot. J. Am. Chem. Soc. 1986, 108, 5641-5642.

(29) Mason, T. J.; Lorimer, J. P. In Sonochemistry, theory, applications and uses of ultrasound in chemistry; Wiley-Interscience: New York, 1989;, pp 252.

(30) Rae, J.; Ashokkumar, M.; Eulaerts, O.; von Sonntag, C.; Reisse, J.; Grieser, F. Estimation of Ultrasound Induced Cavitation Bubble Temperatures in Aqueous Solutions. Ultrason. Sonochem. 2005, 12, $325-329$.

(31) Suslick, K. S.; Flannigan, D. J. Inside a Collapsing Bubble: Sonoluminescence and the Conditions during Cavitation. Annu. Rev. Phys. Chem. 2008, 59, 659-683. 
(32) Hancock, J. In Ultrasonic Cleaning; Cotell, C. M., Sprague, J. A.; Smidt, F. A. J., Eds.; Surface Engineering ASM Handbook, ASM International: 1994; Vol. 5, pp 44-47.

(33) Manikandan, M.; Gopal, J.; Chun, S. Sonophysical Cost Effective Rapid Indigenous Preparation of Aluminium Particles Via Exfoliation of Aluminium Foil. RSC Adv. 2016, 6, 32405-32413.

(34) Kunz, J. N.; Voronine, D. V.; Lu, W.; Liege, Z.; Lee, H. W. H.; Zhang, Z.; Scully, M. O. Aluminum Plasmonic Nanoshielding in Ultraviolet Inactivation of Bacteria. Sci. Rep. 2017, 7, 9026.

(35) Chan, G. H.; Zhao, J.; Schatz, G. C.; Van Duyne, R. P. Localized Surface Plasmon Resonance Spectroscopy of Triangular Aluminum Nanoparticles. J. Phys. Chem. C 2008, 112, 13958-13963. (36) Ramaswamy, A.; Kaste, P. A 'Nanovision" of the Physiochemical Phenomena Occurring in Nanoparticles of Aluminum. J. Energ. Mater. 2005, 23, 1-25.

(37) Lumley, R. In Fundamentals of Aluminium Metallurgy: Production, Processing and Applications; Lumley, R., Ed.; Woodhead Publishing in Materials; Woodhead Publishing: 2011; pp 846.

(38) Gali, O. A.; Shafiei, M.; Hunter, J. A.; Riahi, A. R. The Influence of Work Roll Roughness on the surface/near-Surface Microstructure Evolution of Hot Rolled Aluminum-Magnesium Alloys. J. Mater. Process. Technol. 2016, 237, 331-341.

(39) Wang, J.; Zhou, X.; Thompson, G. E.; Hunter, J. A.; Yuan, Y. Delamination of Near-Surface Layer on Cold Rolled AlFeSi Alloy during Sheet Forming. Mater. Charact. 2015, 99, 109-117.

(40) Kuru, C.; Ipek, S. K.; Dagdelen, E.; Ozsahin, O.; Ulus, A. Material Surface Roughness Change in Twin Roll Casting of Aluminium as Cast Sheet Product. Light Metals 2017 2017, 833-842.

(41) Le, H.; Sutcliffe, M. Analysis of Surface Roughness of ColdRolled Aluminium Foil. Wear 2000, 244, 71-78.

(42) Keles, O.; Dundar, M. Aluminum Foil: Its Typical Quality Problems and their Causes. J. Mater. Process. Technol. 2007, 186, 125137.

(43) Kestens, L. A. I.; Pirgazi, H. Texture Formation in Metal Alloys with Cubic Crystal Structures. Mater. Sci. Technol. 2016, 32, 13031315.

(44) Lu, G.; Kioussis, N.; Bulatov, V.; Kaxiras, E. GeneralizedStacking-Fault Energy Surface and Dislocation Properties of Aluminum. Phys. Rev. B: Condens. Matter Mater. Phys. 2000, 62, 3099-3108.

(45) Muzyk, M.; Pakiela, Z.; Kurzydlowski, K. J. Generalized Stacking Fault Energies of Aluminum Alloys-Density Functional Theory Calculations. Metals 2018, 8, 823.

(46) Lee, S.; Inagaki, H.; Utsunomiya, H.; Saito, Y.; Sakai, T. Ultra Grain Refinement of Commercial Purity Aluminum by a Multi-Stack ARB Process. Mater. Trans. 2003, 44, 1376-1381.

(47) Holscher, M.; Raabe, D.; Lucke, K. Relationship between Rolling Textures and Shear Textures in FCC and BCC Metals. Acta Metall. Mater. 1994, 42, 879-886.

(48) Guilhem, Y.; Basseville, S.; Curtit, F.; Stephan, J.; Cailletaud, G. Numerical Investigations of the Free Surface Effect in ThreeDimensional Polycrystalline Aggregates. Comput. Mater. Sci. 2013, $70,150-162$.

(49) Kashihara, K.; Ikushima, W.; Miyajima, Y.; Terada, D.; Tsuji, N. Change in Crystal Orientations of a $\{100\}$ Pure Aluminum Single Crystal during Accumulative Roll Bonding. Mater. Trans. 2011, 52, 825-829.

(50) Samajdar, I.; Verlinden, B.; Rabet, L.; Van Houtte, P. Recrystallization Texture in a Cold Rolled Commercial Purity Aluminum: On the Plausible Macro- and Micro-Mechanisms. Mater. Sci. Eng., A 1999, 266, 146-154.

(51) Juul Jensen, D., Ed.; In Annealing Textures; Buschow, K. H. J., Cahn, R. W., Flemings, M. C., Ilschner, B., Kramer, E. J., Mahajan, S.; Veyssière, P., Eds.; Encyclopedia of Materials: Science and Technology; Elsevier, Oxford, 2001;, pp 319-323.

(52) Patra, A.; Bates, J. E.; Sun, J.; Perdew, J. P. Properties of Real Metallic Surfaces: Effects of Density Functional Semilocality and Van Der Waals Nonlocality. Proc. Natl. Acad. Sci. U. S. A. 2017, 114, E9188-E9196.
(53) Jian-Min, Z.; Fei, M.; Ke-Wei, X. Calculation of the Surface Energy of Fcc Metals with Modified Embedded-Atom Method. Chin. Phys. 2004, 13, 1082-1090.

(54) Ponja, S. D.; Parkin, I. P.; Carmalt, C. J. Synthesis and Material Characterization of Amorphous and Crystalline (Alpha-) $\mathrm{Al}_{2} \mathrm{O}_{3}$ Via Aerosol Assisted Chemical Vapour Deposition. RSC Adv. 2016, 6, 102956-102960.

(55) Naumenko, D.; Stolzer, L.; Quick, A. S.; Abt, D.; Wegener, M.; Barner-Kowollik, C.; Dal Zilio, S.; Marmiroli, B.; Amenitsch, H.; Fruk, L.; Lazzarino, M. Design of Broadband SERS Substrates by the LaserInduced Aggregation of Gold Nanoparticles. J. Mater. Chem. C 2016, 4, 6152-6159.

(56) Sharifi, N.; Dabirian, A.; Danaei, D.; Taghavinia, N. Aggregates of Plasmonic Nanoparticles for Broadband Light Trapping in DyeSensitized Solar Cells. J. Opt. 2016, 18, 015902.

(57) Yang, Y.; Hu, Y.; Du, H.; Wang, H. Intracellular Gold Nanoparticle Aggregation and their Potential Applications in Photodynamic Therapy. Chem. Commun. 2014, 50, 7287-7290.

(58) Jha, S. K.; Mojarad, N.; Agio, M.; Loeffler, J. F.; Ekinci, Y. Enhancement of the Intrinsic Fluorescence of Adenine using Aluminum Nanoparticle Arrays. Opt. Express 2015, 23, 24719-24729.

(59) Mampallil, D.; Eral, H. B. A Review on Suppression and Utilization of the Coffee-Ring Effect. Adv. Colloid Interface Sci. 2018, $252,38-54$.

(60) Xu, J.; Du, J.; Jing, C.; Zhang, Y.; Cui, J. Facile Detection of Polycyclic Aromatic Hydrocarbons by a Surface-Enhanced Raman Scattering Sensor Based on the Au Coffee Ring Effect. ACS Appl. Mater. Interfaces 2014, 6, 6891-6897.

(61) Yang, S.; Dai, X.; Stogin, B. B.; Wong, T. Ultrasensitive SurfaceEnhanced Raman Scattering Detection in Common Fluids. Proc. Natl. Acad. Sci. U. S. A. 2016, 113, 268-273.

(62) Kumari, G.; Kandula, J.; Narayana, C. How Far can we Probe by SERS? J. Phys. Chem. C 2015, 119, 20057-20064.

(63) Le Ru, E. C.; Blackie, E.; Meyer, M.; Etchegoin, P. G. Surface Enhanced Raman Scattering Enhancement Factors: A Comprehensive Study. J. Phys. Chem. C 2007, 111, 13794-13803.

(64) Pavel, I. E.; Alnajjar, K. S.; Monahan, J. L.; Stahler, A.; Hunter, N. E.; Weaver, K. M.; Baker, J. D.; Meyerhoefer, A. J.; Dolson, D. A. Estimating the Analytical and Surface Enhancement Factors in Surface-Enhanced Raman Scattering (SERS): A Novel Physical Chemistry and Nanotechnology Laboratory Experiment. J. Chem. Educ. 2012, 89, 286-290.

(65) Bell, S. E. J.; Charron, G.; Cortes, E.; Kneipp, J.; Chapelle, M. L. d. l.; Langer, J.; Prochazka, M.; Tran, V.; Schluecker, S. Towards Reliable and Quantitative Surface-Enhanced Raman Scattering (SERS): From Key Parameters to Good Analytical Practice. Angew. Chem., Int. Ed. 2020, 59, 5454-5462.

(66) MacKenzie, M.; Chi, H.; Varma, M.; Pal, P.; Kar, A.; Paterson, L. Femtosecond Laser Fabrication of Silver Nanostructures on Glass for Surface Enhanced Raman Spectroscopy. Sci. Rep. 2020, 10, 17058.

(67) Sharma, B.; Cardinal, M. F.; Ross, M. B.; Zrimsek, A. B.; Bykov, S. V.; Punihaole, D.; Asher, S. A.; Schatz, G. C.; Van Duyne, R. P. Aluminum Film-Over-Nanosphere Substrates for Deep-UV SurfaceEnhanced Resonance Raman Spectroscopy. Nano Lett. 2016, 16, $7968-7973$.

(68) Pisarek, M.; Holdynski, M.; Roguska, A.; Kudelski, A.; JanikCzachor, $\mathrm{M}$. $\mathrm{TiO}_{2}$ and $\mathrm{Al}_{2} \mathrm{O}_{3}$ Nanoporous Oxide Layers Decorated with Silver Nanoparticles-Active Substrates for SERS Measurements. J. Solid State Electrochem. 2014, 18, 3099-3109.

(69) Freeman, L. M.; Pang, L.; Fainman, Y. Maximizing the Electromagnetic and Chemical Resonances of Surface-Enhanced Raman Scattering for Nucleic Acids. ACS Nano 2014, 8, 8383-8391.

(70) Dieringer, J.; McFarland, A.; Shah, N.; Stuart, D.; Whitney, A.; Yonzon, C.; Young, M.; Zhang, X.; Van Duyne, R. Surface Enhanced Raman Spectroscopy: New Materials, Concepts, Characterization Tools, and Applications. Faraday Discuss. 2006, 132, 9-26.

(71) Ross, M. B.; Schatz, G. C. Aluminum and Indium Plasmonic Nanoantennas in the Ultraviolet. J. Phys. Chem. C 2014, 118, 1250612514. 
(72) Hobbs, R. G.; Manfrinato, V. R.; Yang, Y.; Goodman, S. A.; Zhang, L.; Stach, E. A.; Berggren, K. K. High-Energy Surface and Volume Plasmons in Nanopatterned Sub-10 Nm Aluminum Nanostructures. Nano Lett. 2016, 16, 4149-4157.

(73) Swartz, M.; Rodriguez, M.; Quast, A. D.; Cooper, C. T.; Blair, S.; Shumaker-Parry, J. S. Aluminum Nanocrescent Plasmonic Antennas Fabricated by Copper Mask Nanosphere Template Lithography. J. Phys. Chem. C 2016, 120, 20597-20603.

(74) Zhou, L.; Zhang, C.; McClain, M. J.; Manavacas, A.; Krauter, C. M.; Tian, S.; Berg, F.; Everitt, H. O.; Carter, E. A.; Nordlander, P.; Halas, N. J. Aluminum Nanocrystals as a Plasmonic Photocatalyst for Hydrogen Dissociation. Nano Lett. 2016, 16, 1478-1484.

(75) Maidecchi, G.; Duc, C. V.; Buzio, R.; Gerbi, A.; Gemme, G.; Canepa, M.; Bisio, F. Electronic Structure of Core-Shell Metal/Oxide Aluminum Nanoparticles. J. Phys. Chem. C 2015, 119, 26719-26725.

(76) Renard, D.; Tian, S.; Ahmadivand, A.; DeSantis, C. J.; Clark, B. D.; Nordlander, P.; Halas, N. J. Polydopamine-Stabilized Aluminum Nanocrystals: Aqueous Stability and Benzo[a]Pyrene Detection. ACS Nano 2019, 13, 3117-3124. 